\title{
Entangled States and Observables in Open Quantum Relativity
}

\author{
Salvatore Capozziello ${ }^{1,2^{*}}$, Giuseppe Basini ${ }^{3,4}$ \\ ${ }^{1}$ Dipartimento di Scienze Fisiche, Università di Napoli "Federico II" \\ ${ }^{2}$ INFN, Sez. di Napoli, Compl. Univ. di Monte S. Angelo, Edificio G, via Cinthia, I-80126-Napoli, Italy \\ ${ }^{3} \mathrm{CERN}, \mathrm{CH}-1211$ Geneva 23, Switzerland \\ ${ }^{4}$ Laboratori Nazionali di Frascati, INFN via E. Fermi, Frascati I-00100, Roma, Italy \\ E-mail: *capozziello@na.infn.it \\ Received August 17, 2010; revised October 17, 2010; accepted October 25, 2010
}

\begin{abstract}
In the framework of the so called Open Quantum Relativity, we investigate a quantum universe, starting from a minimal set of variables defining the given quantum state. Entanglement between quantum states is the way to link different regions of the universe, even if (apparently) causally disconnected. As a consequence, the concept of causality results recovered and enlarged. Besides, the observed $\Lambda$ CDM model emerges from this picture, giving the possibility to realize a statistical and quantum interpretation of the cosmological constant. In particular, the novelty consists in the fact that the presently observed universe could be the result of several entanglement phenomena giving rise to a certain amount of entropy directly related to the value of cosmological constant.
\end{abstract}

Keywords: Quantum Mechanics, Quantum Cosmology, Entanglement

\section{Introduction}

Up to now, several difficulties occurred in the attempts of quantization of gravity [1-3]. Many authors have discussed the idea that gravity cannot be quantized, due to these problems; other authors, that present models are effective picture and need to be generalized. All the approaches pass through the problems of quantizing General Relativity (GR). In other words, the common approach is to start from a classical field theory to a quantum one. The question is, if it is possible not to pass through a field theory, at the beginning, and to consider, so, only a quantum description of universe, i.e. without a second quantization, where the chosen quantum state has to be found by the use of observable quantities, with minimal choice of the relevant elements of the set too. Then, at the end of a quantum picture, considering also the possibility to have a second quantization, from the quantum picture. This is a peculiar characteristic of the so-called Open Quantum Relativity [4-6]. Such a theory [4] is based on a dynamical unification scheme of fundamental interactions achieved by assuming a 5D space which allows that the conservation laws are always and absolutely valid as a natural necessity. What we usually perceive as violations of conservation laws can be described by a process of embedding and dimensional reduction, which gives rise to an induced-matter theory in the $4 \mathrm{D}$ space-time by which the usual masses, spins and charges of particles, naturally spring out. At the same time, it is possible to build up a covariant symplectic structure directly related to general conservation laws $[7,8]$. Finally, the theory leads to a dynamical explanation of several paradoxes of modern physics (e.g. entanglement of quantum states, quantum teleportation, gamma ray bursts origin, black hole singularities, cosmic primary antimatter absence and a self-consistent fit of all the recently observed cosmological parameters [4,9-12]). A fundamental role in this approach is the link between the geodesic structure and the field equations of the theory before and after the dimensional reduction process. The emergence of an Extra Force term in the reduction process and the possibility to recover the masses of particles, allow to reinterpret the Equivalence Principle as a dynamical consequence which naturally "selects" geodesics from metric structure and vice-versa the metric structure from the geodesics $[6,13]$.

To start with this different point of view, we need a set of minimal number of quantities, corresponding to a set 
of classical objects, observable and measurable. From a theoretical point of view, we have to stress that this is also a new definition of observer. Then the set has to be minimal, in terms of its elements and above all, it has to represent a basis for the cosmological quantum state.

If the set of observable is represented by a certain number of quantities, i.e. $a(t), \Omega_{m, k}, q(t), \ldots$, by the hypothesis of homogeneity and isotropy, i.e. using Friedman-Robertson-Walker (FRW) model, it would be very easy to show that the set could be reduced and a minimal choice would be obtained by considering only two observable among all [14]. The relevant choice is to consider a set based on $\Omega_{m}$ and $\Omega_{k}$, which appears as a minimal one.

The ansatz is that the minimal set is sufficient to implement a quantum state for universe. The minimal choice may allow us to have a state but needs to be rewritten in form of a basis; thanks to Gram-Schmidt procedure we infer this basis; after this, it would be possible to build a general state for universe, whose picture is that the universe would be divided in two space-time regions of interest, characterized by different behaviors of scale factor $a(z)$. GR will match the quantum framework easily after, by considering the definitions of important quantities [15,16], such as $\Omega_{\Lambda}, \Omega_{m}, a(z), \ldots$ which, previously, have been considered independent from the model, in the sense that they only are measurable astrophysical quantities $[17,18]$.

In order to build up a reasonable cosmological entangled state, $|\Psi\rangle \propto \sum_{n}\left|\phi_{n}^{A}\right\rangle\left|\phi_{n}^{B}\right\rangle$, we follow the standard construction of the minimal states by GramSchmidt procedure, as suggested. We find that a

similar state is proportional to $\left|\phi_{i}\right\rangle \equiv\left(\begin{array}{c}\Omega_{m i} \\ \Omega_{k i}\end{array}\right)$. We labeled

the states with $i=1,2$ for defining two regions and we refer to entangled states ansatz hereafter of this work, for stressing the entangled nature of the states. The first step is to have a basis; by Gram-Schmidt is possible using a simplest set of positions for second state

$$
\begin{aligned}
& \Omega_{m 2}^{*}=\Omega_{m 2}-N_{1}^{2}\left(\Omega_{m 1}^{2} \Omega_{m 2}+\Omega_{m 1} \Omega_{k 1} \Omega_{k 2}\right) \rightarrow \Omega_{m 2} \text { and } \\
& \hat{\rho} \equiv p_{2}\left(\begin{array}{l}
N_{2}^{2}\left(\Omega_{m 2}-N_{1}^{2} \Omega_{m 1^{2} m 2}\right)^{2}+\frac{p 1}{p 2} N_{2}^{2} \Omega_{k 2}\left(\Omega_{m 2}-N_{1}^{2} \Omega_{m 1^{2} m 2}\right) \\
N_{2}^{2} \Omega_{k 2}\left(\Omega_{m 2}-N_{1}^{2} \Omega_{m 1^{2} m 2}\right) N_{2}^{2} \Omega_{k 2^{2}}
\end{array}\right)
\end{aligned}
$$

For the sake of simplicity, we assume $\Omega_{k 1} \sim 0$. Moreover the invariant $\operatorname{Tr}(\rho)=1$ reads

$$
\left(-p_{-} \Omega_{m 1}^{6}-p_{+}\left(\Omega_{k 2}^{2}+\Omega_{m 2}^{2}\right) \Omega_{m 1}^{4}+2 p_{+} \Omega_{m 2}^{2} \Omega_{m 1}^{2}-p_{+} \Omega_{m 2}^{2}\right) /\left(\left(\left(\Omega_{m 2}^{2}+\Omega_{k 2}^{2}\right) \Omega_{m 1}^{4}-2 \Omega_{m 2}^{2} \Omega_{m 1}^{2}+\Omega_{m 2}^{2}\right) \Omega_{m 1}^{2}\right)
$$

and the request for $\Omega_{\Lambda}$ gives us

$$
\Omega_{\Lambda}=\frac{\Omega_{m 1^{7}}\left(\Omega_{m 2^{2}}+\Omega_{k 2^{2}}\right)-p 2 \Omega_{m 1^{6}}-2 \Omega_{m 1^{5} 2^{2}}-p_{1} \Omega_{m 1^{4}}\left(\Omega_{m 2^{2}}+\Omega_{k 2^{2}}\right)+p_{1} \Omega_{m 2^{2}}\left(2 \Omega_{m 1^{2}}-1\right)}{\Omega_{m 1^{2}}\left[\Omega_{m 1^{4}}\left(\Omega_{m 2^{2}}+\Omega_{k 2^{2}}\right)+\Omega_{m 2^{2}}\left(1-2 \Omega_{m 1^{2}}\right)\right]}
$$


written with the physical, matter dominance hypothesis, i.e. $\Omega_{m i} \gg \Omega_{k j}$. Note that the trace and $\Omega_{\Lambda}$ are written with simplest positions and the rule $\Omega_{k^{n} j^{m}} \equiv \Omega_{k^{n}}^{{ }^{m}} \Omega_{j}^{m}$ holds.

The eigenvalues of density matrix are(3) and their expressions allows to write a form of entropy for universe as follows.

$$
S=-\lambda_{-} \ln \lambda_{-}-\lambda_{+} \ln \lambda_{+},
$$

which derives from the definition of the so-called Von Neumann entropy [19] $S=-\operatorname{Tr}(\hat{\rho} \ln \hat{\rho})$. This point will be explicitly discussed in the conclusions.

Mixed states deal, so, with the possibility to have a correlation between $\Omega_{\Lambda}$ and the observable $\Omega_{m, k}$, but it appears, in standard approach, not to be physical, because we imagine that such a state in order to have physical meaning, must have properties of entanglement, necessity which is induced by the singularity. In other words, at the Big Bang, in fact, the possible existence of a wave function of the universe, suggests that the wave function of universe now is derived from it but the derivation is allowed if, in the conditions of Big Bang, the wave function is entangled.

Then it is the singularity which implies entangled properties of the state of universe and, as quoted, we can imagine that from the original state comes out from a family of sub-states, each of them derived from the first one and each of them able to explain features of the modern universe, such as structure formations, inflation and so on. This means that entanglement is the key to infer how the correlations of the universe are well-described by the model, while a mixed state describes the reasons for what it is possible to write down expressions of measurable quantities in terms of others. This means that states apparently causally disconnected (or considered in this way up to now) are always quantistically connected.

\section{Properties of Entangled States}

Mixed states are physical observables by which it is possible to build up the functional dependence $\Omega_{\Lambda}=\Omega_{\Lambda}\left(\Omega_{m, k}\right)$, but if we need an universe in which all the regions of it are, among them, connected by a causality principle, we must have a phenomenon of entanglement as the genera. In fact, thel structure of the universe. definition of entanglement, which derives from the superposition principle, suggests us the non-separability of the state and, in particular, the non-factorizability of the state into a singular product.

To have a suitable theory of the universe state $|\Psi\rangle$, we need a basis, from the minimal choice of the number of elements of the state, as explained in the previous section.

Then, we are able to infer the density matrix $\hat{\rho}$, from its definition, concerning the given state, see (5).

Together with this, we used the conditions $p_{+}+p_{-}=1$ of normalized probability and the trace $\operatorname{Tr} \hat{\rho}=1$, together with the two space-time eras $\Omega_{m 1}+\Omega_{k 1}+\sum_{i} \Omega_{X 1 i}=1$ and $\Omega_{m 2}+\Omega_{k 2}+\sum_{j} \Omega_{X 2 j}=1$. In other words, the system also if evolving, is conserving the energy.

Entropy then becomes

$$
S=-\lambda_{3} \ln \lambda_{3}-\lambda_{4} \ln \lambda_{4},
$$

$$
\begin{aligned}
& \lambda_{ \pm}=\frac{1}{2} p_{1} N_{1}^{2} \Omega_{m 1}^{2}+\frac{1}{2} p_{2} N_{2}^{2}\left(\Omega_{k 2}^{2}+\Omega_{m 2}^{2}\right) \pm \\
& \pm \frac{\sqrt{p_{2}^{2} N_{2}^{4} \Omega_{m 2^{4}}+\left(2 N_{2}^{4} \Omega_{k 2^{2}} p_{2}^{2}+2 p_{1} N_{1}^{2} \Omega_{m 1^{2}} p_{2} N_{2}^{2}\right) \Omega_{m 2^{2}}+p_{2}^{2} N_{2}^{4} \Omega_{k 2^{4}}-2 p_{2} N_{2}^{2} \Omega_{k 2^{2}} p_{1} N_{1}^{2} \Omega_{m 1^{2}}+p_{1}^{2} N_{1}^{4} \Omega_{m 1^{4}}}}{2}, \\
& \hat{\rho} \equiv|\alpha|^{2} N_{1}^{2}\left(\begin{array}{cccc}
\Omega_{m 1^{4}} & \Omega_{m 1^{3} k 1} & \Omega_{m 1^{3} k 1} & \Omega_{m 1^{2} k 1^{2}} \\
\Omega_{m 1^{3} k 1} & \Omega_{m 1^{2} k 1^{2}} & \Omega_{m 1^{2} k 1^{2}} & \Omega_{m 1 k 1^{3}} \\
\Omega_{m 1^{3} k 1} & \Omega_{m 1^{2} k 1^{2}} & \Omega_{m 1^{2} k 1^{2}} & \Omega_{m 1 k 1^{3}} \\
\Omega_{k 1^{2} m 1^{2}} & \Omega_{k 1^{3} m 1} & \Omega_{k 1^{3} m 1} & \Omega_{k 1^{4}}
\end{array}\right)+|\beta|^{2} N_{2}^{2}\left(\begin{array}{cccc}
\Omega_{m 2^{4}} & \Omega_{m 2^{3} k 2} & \Omega_{m 2^{3} k 2} & \Omega_{m 2^{2} k 2^{2}} \\
\Omega_{m 2^{3} k 2} & \Omega_{m 2^{2} k 2^{2}} & \Omega_{m 2^{2} k 2^{2}} & \Omega_{m 2 k 2^{3}} \\
\Omega_{m 2^{3} k 2} & \Omega_{m 2^{2} k 2^{2}} & \Omega_{m 2^{2} k 2^{2}} & \Omega_{m 2 k 2^{3}} \\
\Omega_{k 2^{2} m 2^{2}} & \Omega_{k 2^{3} m 2} & \Omega_{k 2^{3} m 2} & \Omega_{k 2^{4}}
\end{array}\right)+ \\
& +\left(\alpha \beta^{*}+\beta \alpha^{*}\right)\left(p_{+}-p_{-}\right) N_{1} N_{2}\left(\begin{array}{cccc}
\Omega_{m 1^{2} m 2^{2}} & \Omega_{m 1^{2} m 2 k 2} & \Omega_{m 1^{2} m 2 k 2} & \Omega_{m 1^{2} k 2^{2}} \\
\Omega_{m 1 k 1 m 2^{2}} & \Omega_{m 1 k 1 m 2 k 2} & \Omega_{m 1 k 1 m 2 k 2} & \Omega_{m 1 k 1 k 2^{2}} \\
\Omega_{m 1 k 1 m 2^{2}} & \Omega_{m 1 k 1 m 2 k 2} & \Omega_{m 1 k 1 m 2 k 2} & \Omega_{m 1 k 1 k 2^{2}} \\
\Omega_{k 1^{2} m 2^{2}} & \Omega_{k 1^{2} m 2 k 2} & \Omega_{k 1^{2}{ }^{2} k 2} & \Omega_{k 1^{2} k 2^{2}}
\end{array}\right) \text {. }
\end{aligned}
$$


because two of the four eigenvalues are zero, i.e. $\lambda_{1,2}=0$ and $0 \ln 0 \equiv 0$. The expression, given by mixed states is quite similar for construction, in sense that here we have two eigenvalues different from zero. Then we can imagine a mixed state as a subcase of an entangled state, and this is true, simply by the definition of the mixed entangled density matrix.

\subsection{Mutual Information between Eras}

The basic use of the entropy $S$, inherent to an entangled state, is the role of measurement of information and of correlation between two regions of universe and, it is surely, referred to the possible expression of entropy for universe. The thermal entropy would really derive, so, from the quantum expression of Von Neumann entropy, inherent to the quantum picture proposed. In the simplest ansatz we adopted (two regions of different space-time epoches) we can note that, in general, the Von Neuman entropy of Equation (6) obeys to the inequality

$$
S(\rho) \leq S\left(\rho^{A}\right)+S\left(\rho^{B}\right),
$$

where $\rho=\rho^{A B}$. From that property of $S$, it is possible to find the so-called mutual information [19] as follows:

$$
I\left(\hat{\rho}^{A} \| \hat{\rho}^{B}\right)=S\left(\hat{\rho}^{A}\right)+S\left(\hat{\rho}^{B}\right)-S(\hat{\rho}) .
$$

This relation represents the superposition of the two regions entropies, that is to say that we have to imagine that an universe composed by two eras, or regions, each of them having an its own entropy value, identified by the reduced density matrix $\hat{\rho}^{A}$, for first era and, otherwise, by $\hat{\rho}^{B}$, for the other one. However, this scheme can be extended to any number or regions.

The amount of "intersection" between these eras, by considering entropy, is quantified by the mutual information, which appears to be regular and, so, simply by the value of it, we infer how great is the correlation between regions.

The mutual information for our purpose is

$$
I\left(\hat{\rho}^{A B}\right)=\frac{\ln \Omega_{m 1}^{2}}{\Omega_{m 1}^{2}}+\frac{\ln \left(\frac{\Omega_{m 1}^{2}-1}{\Omega_{m 1}}\right)^{2}}{\left(\frac{\Omega_{m 1}^{2}-1}{\Omega_{m 1}}\right)^{2}},
$$

in the case of spatial curvature parameter density $\Omega_{k 1} \ll 1$ and $\Omega_{k 2} \ll 1$ and $\Omega_{m 2} \approx \Omega_{m 1}$, which has to be considered the case of matter dominance and maximally entangled condition, i.e. $\alpha=\beta=\frac{1}{\sqrt{2}}$. In the case of no maximally entangled states we have

$$
I\left(\hat{\rho}^{A B}\right)=\frac{\ln \Omega_{m 1}^{2}}{\Omega_{m 1}^{2}}+\frac{\ln \left(\frac{\Omega_{m 1}^{2}-1}{\Omega_{m 1}}\right)^{2}}{\left(\frac{\Omega_{m 1}^{2}-1}{\Omega_{m 1}}\right)^{2}}+f(\alpha, \beta),
$$

with $f(\alpha, \beta)=\left(\alpha^{2}+\beta^{2}\right) \ln \left(\alpha^{2}+\beta^{2}\right)$ which represents the treshold of the entanglement process. Both cases are evaluated in the simplest hypothesis of $p_{-}=\frac{1}{2}$. The case of curvature dominance is specular to the case of matter dominance, simply changing in the formula of Equation (9) $\Omega_{m 1}$ with $\Omega_{k 1}$.

The effect of curvature is that of shifting the value of mutual information, changing its strength.

\section{Conclusions}

Entanglement suggests how to construct a quantum state for the universe using the theoretical tools of Open Quantum Relativity, i.e. considering a quantization procedure at the very foundation of the theory.

In this way, GR is recovered from Entanglement and it is described $a$ t the end of the formulation of $\Omega_{\Lambda}$, in order to understand the role of $\Lambda$ and of other objects [14] in cosmology. This generality is a peculiar feature of Open Quantum Relativity [5].

The final sense of this procedure is to find a definition of a quantum state in terms of minimal choice of observable quantities. The way to take into account the state is the construction of entangled states. This allows us to explain the reasons why $\Omega_{\Lambda}$ term appears to be constant in time and avoids the coincidence problem [20]. $\Omega_{\Lambda}$ would become, therefore, a statistical quantum

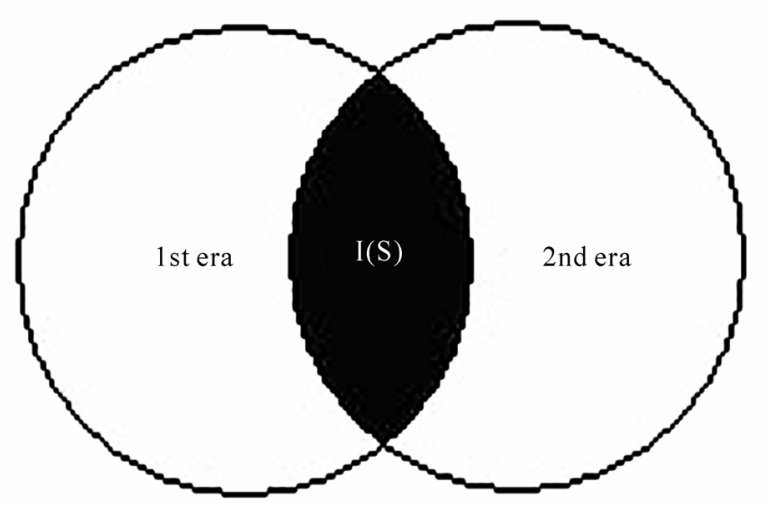

Figure 1. In this graphic is plotted a scheme for understanding the exact role of mutual information $I(S)$, in the context of two quantum regions. The degree of superposition of two regions is directly proportional to $I(S)$. 
result. A mixed state allows us to understand why $\Omega_{\Lambda}$ is dependent from other densities (matter and curvature) and so it is a derived emergent quantity, not a fundamental one.

These results can be framed in a canonical quantization scheme recovering standard results of quantum cosmology. The procedure has been proposed in [13], and describes a wave function, written as $\phi \equiv\langle x \mid \Psi\rangle$, where the state $|\Psi\rangle$ is the entangled one, so a second quantization would be expressed as

$$
\Psi(x)=\sum_{n=0}^{\infty} e^{-I(a, \phi, N)} D a D \phi D N,
$$

where $I$ is the action. The form of the action allows also to write down, in the semiclassical approach, the wave function of the universe as

$$
\Psi \sim \exp \{-I(a, \phi)\} .
$$

As concluding remark, we can say that Open Quantum Relativity is at the crossing point between GR and Quantum Mechanics, thanks to the fact that it generalizes the causality principle (including entanglement). The question if a physical system can be considered existing either with or without information is solved in the sense that the physical system without information is not interacting with systems outside of it. This means that the solutions (10) and (11) with real values are what we are ordinarily experiencing while the immaginary solutions (the instantons) are not producing observable physical effects unless we connect them by entanglement. The emergence of "entropy", in the usual sense, suggests to take into account only one time arrow until we do not take into account immaginary solutions and the entanglement phenomenon. In such a case a backward dynamics (and then two time arrows) have to be considered.

\section{References}

[1] J. B. Hartle, "The Quantum Structure of Space and Time," Proceedings of the 23rd Solvay Conference, Singapore, 2007, pp. 21-43.

[2] J. J. Halliwell and J. B. Hartle, "Integration Contours for the No-Boundary Wave Function of the Universe," Physical Review D, Vol. 41, No. 6, 1990, pp. 1815-1834.

[3] J. B. Hartle, "Prediction in Quantum Cosmology,” In: S. Carter and J. B. Hartle, Eds., Gravitation in Astrophysics, Cargese, 1986, pp. 392-360.

[4] G. Basini and S. Capozziello, "A Dynamical Unification Scheme from General Conservation Laws,” General Relativity and Gravitation, Vol. 35, No. 12, 2003, pp. 22172248.
[5] G. Basini and S. Capozziello, "Quantum Mechanics, Relativity and Time,” General Relativity and Gravitation, Vol. 37, No. 1, 2005, pp. 115-165.

[6] G. Basini and S. Capozziello, "The Spacetime Structure of Open Quantum Relativity,” Progress in Physics, Vol. 3, 2007, pp. 36-41.

[7] G. Basini and S. Capozziello, "A General Covariant Symplectic Structure from Conservation Laws," Modern Physics Letters A, Vol. 20, No. 4, 2005, pp. 251-262.

[8] G. Basini and S. Capozziello, “A General Covariant Symplectic Structure for Gravitational, Electromagnetic and Dirac Fields,” International Journal of Modern Physics D, Vol. 15, No. 4, 2006, pp. 583-602.

[9] G. Basini and S. Capozziello, "Conservation Laws, Causality, Entanglement and Topology Changes. A Gate for a Time Machine,” European Physical Journal, Vol. 63, 2003, pp. 166-172.

[10] G. Basini, S. Capozziello and G. Longo, "The General Conservation Principle. Absolute Validity of Conservation Laws and Their Role as Source of Entanglement, Topology Changes, and Generation of Masses,” Physical Letters A, Vol. 311, No. 6, 2003, pp. 465-473.

[11] G. Basini, S. Capozziello and G. Longo, "Gamma Ray Bursts as a Signature for Entangled Gravitational Systems," Astroparticle Physics, Vol. 20, No. 4, 2004, pp. 457-466.

[12] G. Basini, F. Bongiorno and S. Capozziello, "Singularity Free Solutions from Scalar-Tensor Gravity Compared with Recent Cosmological Observations,” International Journal of Modern Physics D, Vol. 13, No. 4, 2004, pp. 717-737.

[13] G. Basini and S. Capozziello, "Multi-Spaces and Many Worlds from Conservation Laws," Progress in Physics, Vol. 4, 2006, pp. 65-72.

[14] P. J. E. Peebles, "Principles of Physical Cosmology," Princeton University Press, Princeton, 1993.

[15] R. M. Wald, "General Relativity," The University of Chicago, Chicago, 1984.

[16] C. W. Misner, K. S. Thorne and J. A. Wheeler, "Gravitation,” Freeman and Company, New York, 1973.

[17] A. G. Riess, et al., "Type Ia Supernova Discoveries at z > 1 from the Hubble Space Telescope: Evidence for Past Deceleration and Constraints on Dark Energy Evolution,” Astrophysical Journal, Vol. 607, No. 2, 2004, pp. 665687.

[18] R. Rebolo, et al., "Cosmological parameter estimation using Very Small Array data out to $\mathrm{l}=1500$," MNRAS, Vol. 353, No. 3, 2004, pp. 747-759.

[19] M. A. Nielsen and I. L. Chuang, "Quantum Computation and Quantum Information,” Cambridge University Press, Cambridge, 2002.

[20] S. Weinberg, “The Cosmological Constant Problem,” Reviews of Modern Physics, Vol. 61, No. 1, 1989, pp. 1-23. 